La revue La revue pour l'histoire du CNRS

POUR L'HISTOIRE DU CNRS

$22 \mid 2008$

La géopolitique mode d'emploi

La géopolitique : une histoire contrastée

Yves Lacoste

(2) OpenEdition

Journals

Édition électronique

URL : https://journals.openedition.org/histoire-cnrs/8082

DOI : 10.4000/histoire-cnrs.8082

ISSN : 1955-2408

Éditeur

CNRS Éditions

Édition imprimée

Date de publication : 3 octobre 2008

ISBN : 978-2-271-06676-3

ISSN : 1298-9800

Référence électronique

Yves Lacoste, "La géopolitique : une histoire contrastée », La revue pour l'histoire du CNRS [En ligne]

22 | 2008, mis en ligne le 03 octobre 2010, consulté le 20 mai 2021. URL : http://

journals.openedition.org/histoire-cnrs/8082 ; DOI : https://doi.org/10.4000/histoire-cnrs.8082

Ce document a été généré automatiquement le 20 mai 2021.

Comité pour l'histoire du CNRS 


\title{
La géopolitique : une histoire contrastée
}

\author{
Yves Lacoste
}

1 Les conflits pour des territoires existent depuis des millénaires (et d'abord pour des territoires de chasse), entre des tribus, des cités, des empires, des peuples et des nations. Quelques-unes de ces guerres ont chacune fait l'objet de récits historiques écrits par des chefs de guerre victorieux (César, La guerre des Gaules) ou des témoins privilégiés (Thucydide et l'Histoire de la Guerre du Péloponnèse). Mais nous dirons qu'il faut attendre le XIX siècle, pour que ces conflits pour du territoire ou des étendues marines commencent à faire l'objet d'études comparatives et systématiques qui prennent en compte non seulement les forces en présence, mais surtout les multiples caractéristiques géographiques des espaces disputés.

2 Certes la géographie (étymologiquement, dessiner la terre et d'abord des cartes) est un des tout premiers savoirs scientifiques. Il a 2500 ans d'existence, depuis Hérodote et ses Enquêtes et il s'est étendu et précisé au cours des siècles au fur et à mesure des mesures et des observations faites par les conquérants, les navigateurs et les explorateurs. C'est seulement au début du XIX siècle, après les guerres napoléoniennes, et tout d'abord en Prusse, que la géographie commence à faire l'objet d'un enseignement destiné non plus seulement aux chefs de guerre, mais aussi à des milieux sociaux de plus en plus larges (et même aux élèves des écoles) et qu'elle devient - d'abord à l'université de Berlin discipline universitaire pour former des professeurs de lycée; tout ceci n'est pas sans rapport avec le mouvement pour l'unité allemande. Cette géographie universitaire qui produit des classifications et des outillages intellectuels, se compose progressivement de secteurs de plus en plus nombreux : géographie des formes du relief, géographie des climats, de la végétation... de la population, géographie économique, géographie politique. C'est à partir de ce dernier secteur que va se constituer la géopolitique.

Première phase : la géopolitique allemande, 1890-1945

3 En 1887, le grand géographe Friedrich Ratzel qui est déjà l'auteur d'une vaste Anthropogéographie (1882), publie en 1887 une Politische Geographie. C'est par la contraction de ces deux termes, sous la plume de Rudolf Kjellen, un juriste suédois 
(germanophile) devenu géographe, qu'est apparu quelques temps plus tard le mot Geopolitik. Cette géopolitique allemande s'est abusivement réclamée des thèses biologiques de Darwin, en prétendant qu'entre les peuples, existait une sélection naturelle, facteur de progrès, comparable à celle qui existe entre les espèces animales. Ce discours pseudo-scientifique connut un succès d'autant plus grand en Allemagne qu'il fournissait une commode «justification» scientifique à l'expansion pangermaniste. Aussi le terme de géopolitique ne fut guère utilisé par l'École géographique française qui s'était développée fin XIXe, à l'exemple de l'École géographique allemande.

4 Fort prisé dans les milieux dirigeants du Reich à la veille de la première guerre mondiale, le mot Geopolitik, après la défaite de 1918, trouva, par l'entremise des professeurs de lycée, un large écho dans l'opinion allemande pour la pousser à dénoncer les injustices du Traité de Versailles et préparer la revanche.

5 La géopolitique fut célébrée par les nazis comme « la science allemande » et ceux-ci en firent la justification prétendument scientifique des besoins d'»espace vital» du peuple allemand. La géopolitique fut aussi le grand argument du stupéfiant pacte germano-soviétique d'août 1939, Staline s'étant laissé séduire par l'idée d'un pacte continental, dont la première étape fut le partage de la Pologne. La gravité de cette erreur stratégique apparut aux Soviétiques en juin 1941, lorsque l'armée du Reich n'ayant alors plus rien à craindre à l'ouest (après la défaite française de juin 1940) se lança brusquement à la conquête de l'Union soviétique. Les SS entreprirent l'extermination des populations juives et slaves des territoires du futur Grand Reich, la génétique ayant été invoquée pour affirmer qu'il s'agissait de races inférieures ou impures.

Le mot géopolitique frappé d'interdit et cependant banalisé 1945-198...

6 Aussi dès la fin de la seconde guerre mondiale, toute allusion à la géopolitique, jugée alors théorie spécifiquement nazie, fut proscrite dans tous les pays européens et aux États-Unis. Ensuite, durant des décennies, il fut moralement interdit de parler de géopolitique à propos de toutes questions relatives à des territoires, qu'il s'agisse des accords de Yalta, de la division de l'Allemagne et de ses nouvelles frontières, ou celles de la Pologne, de la partition de l'Inde après l'indépendance, de la Corée ou du Vietnam en 1954, etc. En URSS comme dans d'autres États « socialistes », ce fut un crime grave durant des décennies que de parler de géopolitique et la géographie humaine y fut même interdite par crainte de dérive géopolitique. En France, le mot géopolitique resta moralement proscrit (notamment durant la guerre d'Algérie) sous l'effet d'une stricte vigilance idéologique de gauche dans les milieux intellectuels, sauf quand il servait à diaboliser l'impérialisme américain.

7 Durant des décennies, les souvenirs les plus épouvantables du nazisme ont donc été associés au mot géopolitique, mais si quelques lecteurs en cherchaient alors la signification dans un dictionnaire, le Larousse ou le Robert, les définitions qu'ils y trouvaient, étaient d'une étonnante sérénité naturelle. Le Grand Larousse Universel (1962) définit la géopolitique comme "l'étude des rapports qui unissent les États, leurs politiques et les lois de la nature, ces dernières déterminant les autres». Dans la dernière édition du Petit Larousse 2008, la géopolitique est «l'étude des rapports entre les données géographiques et la politique des États». Pour le Robert, et c'est encore le cas aujourd'hui, la géopolitique «est l'étude des rapports entre les données naturelles de la géographie et la politique des États ». Pas la moindre allusion à l'implication de la géopolitique (ou plus 
exactement de discours géopolitiques d'un certain type) dans les plus grandes tragédies du XXe siècle. Est-ce l'effet d'une règle lexicographique qui serait d'éviter de mettre en cause une forme d'» étude » (sinon de discipline) considérée comme scientifique?

8 Le préfixe "géo " implique la géographie, mais dans ces dictionnaires celle-ci est réduite aux données naturelles, celles-ci étant jugées fondamentales, et ce en dépit des géographes eux-mêmes qui récusent le « déterminisme » des dites données naturelles sur les activités humaines. Ces prétendues définitions de la géopolitique qui perdurent dans de larges milieux intellectuels et qui proclament dans « la politique des États » la place déterminante des " données naturelles ", font curieusement silence sur le rôle de l'histoire. Pourtant son importance est d'évidence considérable dans tout problème géopolitique, ne serait-ce que dans le tracé de toute frontière et plus encore de celles qui sont contestées. Les "données naturelles » sensées déterminer la politique des États, ne changeant que sur des milliers ou des millions d'années et les «lois de la nature " étant éternelles, on devrait être logiquement réduit, en matière de géopolitique, à des lieux communs du genre «l'Angleterre est une île », « le Japon est un archipel » ou l'immensité du territoire de la Russie.

Ce ne sont évidemment pas ce genre de banalités géographiques qui explique le succès croissant du mot géopolitique depuis une vingtaine d'années en France. Succès d'autant plus étonnant que la géographie, réduite à une fastidieuse discipline scolaire, est - à la différence de l'histoire - assez mal vue des intellectuels. Le mot géopolitique est pourtant devenu aujourd'hui un terme à la mode. Il subit depuis peu une véritable inflation sémantique, à telle enseigne par exemple que des philosophes anglo-saxons " postmodernes » séduits par les discours de leurs confrères parisiens, dissertent sur "la géopolitique de la connaissance et de la différence coloniale ».

Le rôle d'Hérodote (1976), revue de géographie et de géopolitique

Pour mieux combattre les raisons pour lesquelles de nos jours, les intellectuels tiennent la géographie en piètre estime, ma fierté de géographe m'avait incité à comprendre les origines et la raison d'être de ce savoir qui, dès ses débuts, fut politiquement fondamental, du moins pour ceux qui ont du pouvoir. Cela me fit découvrir Hérodote, le premier grand géographe autant qu'il fut le premier grand historien. Il y a vingt-cinq siècles, il mena ses Enquêtes dans l'empire perse et jusqu'en Égypte pour que les Grecs sachent mieux aller le combattre et le prendre à revers, quand quelque nouveau Darius lancerait contre eux une troisième guerre médique. Celle-ci n'eut pas lieu, mais un siècle plus tard, Alexandre le Grand sera guidé jusqu'en Égypte et jusqu'en Afghanistan par les Enquêtes du géographe.

11 Comme pendant longtemps j'ai eu la chance, à plusieurs reprises, de mesurer sur le terrain l'efficacité tactique et l'importance stratégique du raisonnement géographique, j'ai souhaité en convaincre les intellectuels comme les futurs professeurs d'histoire/ géographie. Aussi en 1976, grâce au soutien de l'éditeur François Maspero, ai-je lancé la revue Hérodote avec un petit groupe de jeunes chercheurs qui avaient été mes étudiants (à «Vincennes" la fameuse université «soixante-huitarde ») et avec l'appui de quelques-uns de mes amis géographes (et non des moindres). Le sous-titre de la revue fut d'abord Stratégies- Géographies-Idéologies. Et aujourd'hui, trente ans plus tard, c'est encore la principale revue de géographie... et de géopolitique. Hérodote a joué et joue encore un rôle important dans le développement de l'analyse géopolitique en France.

En 1976, j'avais aussi publié (chez Maspero) un petit livre dont le titre fit grand scandale chez les géographes, mais qui suscita l'attention de nombreux lecteurs et aussi 
des journalistes : La géographie, ça sert d'abord à faire la guerre. J'y traitais de la géographie des États-majors et de celle des professeurs, il était déjà question de rivalités de pouvoirs sur des territoires, mais il n'était pas fait mention de géopolitique (sauf pour stigmatiser très brièvement son rôle au service du nazisme). Ce silence traduisait mon peu d'intérêt à l'égard des raisonnements et méthodes de Ratzel, le fondateur de cette géopolitique allemande qui est en vérité tout à fait dépassée.

En effet, dans Politische Geographie, Ratzel ne fait aucune analyse détaillée de situation conflictuelle concrète. Il prétend établir de soi-disant « lois » de la géographie politique et pour cela il énumère, pour prouver la validité de chacune d'elles, de longues séries d'exemples historiques brièvement évoqués et choisis par lui depuis l'Antiquité dans diverses parties de l'Europe et du monde. Ces « lois» (par exemple, est-ce qu'un État qui possède l'aval d'un fleuve, doit en contrôler l'amont ?) avaient en fait pour but de justifier l'expansion du Reich dans telle ou telle situation géographique; les cas qui auraient infirmer ces prétendues « lois » étant passées sous silence.

Il s'agit en vérité d'un discours d'allure axiomatique (néo-darwinien) sur l'inégale capacité biologique des peuples et des races humaines à conquérir et à organiser des territoires de taille très inégale : le Reich, en tant qu'» État jeune », a un grand besoin naturel d'espace (c'est le fameux « espace vital»), il a donc grand droit à l'espace, alors que les «États vieux» (comme la France, bien sûr) occupent abusivement de trop vastes territoires.

15 Voilà pourquoi, en 1976, dans La géographie, ça sert d'abord à faire la guerre, je n'avais pas jugé utile d'évoquer la géopolitique, ce mot que l'on n'utilisait plus depuis des décennies, ne désignant alors qu'une théorie pseudo-géographique sans fondement sérieux et que les nazis avaient utilisée pour pousser le peuple allemand dans une aventure catastrophique.

La brusque réapparition du mot géopolitique en 1979

16 En janvier 1979, le mot géopolitique réapparut brusquement dans la presse, et tout d'abord dans Le Monde, pour désigner un événement tout à fait inattendu et spectaculaire : la guerre qui venait d'éclater, pour une question de frontière, entre les Khmers rouges et le Vietnam communiste. Non seulement ce conflit, si les Américains tentaient d'en profiter, pouvait relancer la guerre de trente ans dans cette partie du monde, mais il pouvait aussi provoquer une troisième guerre mondiale, la Chine communiste attaquant «pour le punir » le Vietnam que soutenait l'Union soviétique. L'enjeu territorial explicite du conflit Vietnam-Cambodge justifiait l'emploi du terme géopolitique et les censeurs se tinrent cois devant ce conflit entre pays communistes. Quelques mois plus tard, le départ des Américains d'Iran, chassés par la révolution de Khomeiny, fut présenté à juste titre dans la presse comme un grand changement géopolitique et l'année 1979 se termina par un changement non moins grand, lui aussi dénommé géopolitique : l'invasion de l'Afghanistan par les Soviétiques dont on pouvait craindre qu'ils foncent vers les «mers chaudes».

17 Le mot géopolitique apparut dans la presse comme un mot neuf, du fait de la longue période d'oubli durant laquelle il avait été proscrit. Mais surtout il ne "collait » pas avec les discours qui depuis des décennies opposaient «monde libre» et «bloc socialiste ». Les journalistes ne savaient pas trop ce que pouvait signifier ce mot de géopolitique et certains pensèrent même qu'il s'agissait d'une science nouvelle. Toujours est-il qu'il provoquait l'attention des lecteurs, surpris de l'importance que pouvaient prendre des données géographiques et inquiets des conséquences 
internationales que pouvaient avoir des rivalités de pouvoirs sur des territoires, même de modestes dimensions. Les journalistes étaient demandeurs d'explications sur ce qu'était géopolitique, mais, parmi les géographes consultés, la plupart d'entre eux, pris de court, rejetèrent dans l'idéologie du nazisme ce terme qu'en vérité, ils ne connaissaient guère. En effet dans la corporation hiérarchisée qu'ils constituent à l'université, il était devenu quasiment de règle (non dite) depuis les débuts du $\mathrm{XX}^{\mathrm{e}}$ siècle (par souci «d'objectivité scientifique»?) d'éviter de traiter les questions de pouvoirs et de territoire.

18 C'est à ces questions que s'intéressait depuis plusieurs années la petite équipe d'Hérodote et ce fut elle qui prit le risque de reprendre, chez les géographes, le terme de géopolitique. À la différence de Ratzel et de ses épigones qui se référaient à de soi-disant lois géopolitiques pour prouver le bien-fondé des visées allemandes sur tel ou tel territoire, en passant sous silence les droits des autres peuples, Hérodote (nom collectif) a posé comme principe de la nouvelle géopolitique qu'il fallait, par souci d'honnêteté et d'efficacité, confronter méthodiquement les points de vue des protagonistes. En effet, chaque portion de territoire que se disputent deux ou plusieurs forces politiques fait l'objet de représentations géopolitiques contradictoires où se mêlent des valeurs symboliques antagonistes et des arguments historiques qui privilégient telle ou telle période de l'histoire pour en passer d'autres sous silence.

La formation progressive de l'École géopolitique française

Le petit groupe d'Hérodote avec l'appui de ses amis, mène depuis trente ans et en fonction de l'actualité, un travail comparatif de réflexion et d'observation portant sur l'analyse précise d'un certain nombre de cas concrets, des situations géopolitiques particulièrement compliquées. En 1986, paraissent, sous ma direction et en fait celle de Béatrice Giblin, les trois tomes de Géopolitiques des régions françaises (Fayard), trente auteurs presque tous géographes montrant ainsi que l'analyse géopolitique ne se limite pas aux conflits entre États et qu'elle est éclairante au sein d'une même nation, du fait des rivalités électorales entre partis politiques. En 1988, Michel Foucher publie Fronts et frontières - Un tour du monde géopolitique ${ }^{1}$. En octobre 1989 débute le premier séminaire de la Formation doctorale de géopolitique, à l'université Paris VIII ("Vincennes" à Saint-Denis). Coïncidence, un mois plus tard tombe le «mur de Berlin». En 1990, l'effondrement des régimes communistes dans les pays d'Europe de l'Est ouvre des perspectives géopolitiques nouvelles et, fin 1991, l'annonce stupéfiante de la dislocation soudaine de l'Union soviétique provoquent en France un redoublement d'intérêt pour toutes les questions géopolitiques, aussi bien dans les milieux de droite que de gauche. Est alors mis en chantier un gros Dictionnaire de géopolitique (Flammarion, 1993-95) sous ma direction, avec une centaine d'auteurs, pour une grande part collaborateurs réguliers d'Hérodote.

Sous l'influence notamment d'Hérodote, dont la fonction critique ne l'écartait pas de projets universitaires, des jeunes gens qui avaient déjà «fait de l'histoire » ou du droit, des sciences politiques ou de la géographie, dans diverses universités, poussèrent leur intérêt pour la géopolitique, en souhaitant y consacrer des années de recherches, afin de soutenir d'abord un DEA puis une thèse en géopolitique, portant sur l'analyse très approfondie d'une situation géopolitique précise. C'est pour les accueillir et les guider qu'en 1989 à l'université Paris VIII, j'ai constitué avec Béatrice Giblin et d'autres collègues, le Centre de recherches et d'analyses géopolitiques qui sera en France pendant quinze ans l'unique Formation doctorale de géopolitique. Elle a accueilli de 
jeunes chercheurs venus de diverses universités françaises et européennes, notamment de Russie et des pays d'Europe médiane. Chaque année, est menée à bien une trentaine de solides mémoires de recherche pour le DEA de géopolitique devenu depuis deux ans un master de recherches et c'est au total une cinquantaine de thèses de doctorat de géopolitique qui ont été soutenues. Chacune d'elles est une étude approfondie d'un espace de conflit et une contribution originale à la réflexion théorique en géopolitique.

Ces succès ont permis en 2002, toujours à l'université Paris VIII et dans le même esprit, la création par Béatrice Giblin de l'Institut français de géopolitique. Celui-ci comprend un master de recherches avec deux spécialités: une spécialité recherche "Géopolitique, enjeux territoriaux et rivalités de pouvoirs" et une spécialité professionnelle "Géopolitique locale, aménagement, gestion et enjeux territoriaux ». Plusieurs thèses de géopolitique y sont soutenues chaque année. Cofondatrice d'Hérodote en 1976, Béatrice Giblin est notamment une spécialiste reconnue de ce que l'on peut appeler la géopolitique interne (Nouvelle géopolitique des régions françaises, 2006). Il existe donc désormais une école française de géopolitique.

Son influence commence à se faire plus ou moins entendre dans diverses universités et surtout dans de nombreuses écoles de commerce où des géographes enseignent désormais la géopolitique. Un master de géopolitique existe depuis peu à l'université Paris I et à l'École normale supérieure (rue d'Ulm) avec la participation de Michel Foucher qui, avant d'être ambassadeur, a dirigé le Centre d'analyse et de prévision du ministère des Affaires étrangères.

23 Ainsi depuis trente ans s'est constitué progressivement, à partir d'Hérodote, un groupe nombreux et relativement cohérent de jeunes spécialistes de géopolitique, (le terme de géopoliticien a la connotation fâcheuse de politicien), ce qui est pour le moment un cas exceptionnel au plan mondial. Toutefois, ils ne sont pas les seuls à analyser des rivalités de pouvoirs étatiques ou nationaux, car il existe en France, comme dans divers pays européens et surtout aux États-Unis, de nombreux centres de recherches sur les « relations internationales ». Cette expression désigne des problèmes très comparables à ceux dont il est question en géopolitique. Cependant, les spécialistes de relations internationales, qu'ils aient une formation d'historien ou surtout de science politique, n'utilisent guère le mot géopolitique. À son encontre, il ne s'agit plus guère du tabou d'antan, mais surtout de leur méconnaissance du raisonnement géographique.

Le champ de la géopolitique, tel que le conçoit l'École géopolitique française est en fait plus large que celui des relations internationales puisque celle-ci prend en compte des conflits locaux, par exemple entre des tribus ou au sein de grandes agglomérations urbaines où il s'agit de conflits entre bandes rivales. Certes l'approche en termes de relations internationales est particulièrement efficace pour l'analyse des rapports diplomatiques, commerciaux, financiers et culturels entre les États, mais la géopolitique est mieux armée pour l'étude des conflits sur " le terrain », en raison de la place qu'elle accorde aux raisonnements géographiques surtout s'ils sont menés à différents niveaux d'analyse spatiale. L'étude d'un grand phénomène politique comme l'islamisme relève tout autant des spécialistes des «relations internationales » que de la géopolitique. 


\section{BIBLIOGRAPHIE}

Lacoste Y. Atlas géopolitique. Larousse, 2007.

Lacoste Y. Géopolitique de la Méditerranée. Armand Colin, 2006.

Lacoste Y. Géopolitique. La longue histoire d'aujourd'hui. Larousse, 2006.

Lacoste Y. De la géopolitique aux paysages. Dictionnaire de la Géographie. Armand Colin, 2003.

Lacoste Y., Monsel P. L'eau des hommes. Le Cercle d'art, 2002.

Lacoste Y. Vive la nation. Destin d'une idée géopolitique. Fayard, 1998.

Lacoste Y. (sous la direction de, 100 auteurs). Dictionnaire de géopolitique. Flammarion, 1995.

\section{NOTES}

1. Fayard, 1991, une nouvelle édition, qui fait toujours autorité.

\section{RÉSUMÉS}

Rivalités de pouvoirs ou d'influences sur des territoires, rivalités pour le contrôle ou la domination de territoires de grande ou de très petite taille, rivalités entre des pouvoirs politiques de toutes sortes, et pas seulement entre des États, mais aussi entre des tribus, des mouvements politiques ou religieux... tel est le sens qu'Yves Lacoste donne au mot géopolitique.

According to Yves Lacoste, the word geopolitics means: power rivalries within territories; competition for the control or the domination of large or very small territories of all sorts. These rivalries occur not only between nation States but also between tribes, political groups or religious forces.

\section{AUTEUR}

\section{YVES LACOSTE}

Yves Lacoste est géographe, spécialiste de géopolitique. Il a fondé en 1989 le Centre de recherches et d'analyses de géopolitiques (aujourd'hui Institut français de géopolitique) et a créé Hérodote, revue de géographie et de géopolitique (trimestrielle publiée par les Éditions La Découverte). 